\title{
Visual-Visual Associative Learning and Reward-Association Learning in Monkeys: The Role of the Amygdala
}

\author{
David Gaffan, E. A. Gaffan, ${ }^{1}$ and Susan Harrison \\ Department of Experimental Psychology, Oxford University, Oxford OX1 3UD, England, and 'Department of Psychology, \\ Reading University, Reading RG6 2AL, England
}

\begin{abstract}
Three Cynomolgus monkeys (Macaca fascicularis) took part in an experiment on visual learning set in an automatic apparatus. Each new visual discrimination problem was solved using a visual secondary reinforcer consisting of a white line. If the monkey chose the correct stimulus (by touching it), the white line appeared over the correct stimulus. Primary food reward was delivered only after a new problem was solved to a criterion, and the problem was then replaced by a new one. Thus, within-problem learning did not rely on primary reinforcement but on the visual secondary reinforcer. The animals were trained preoperatively in visual learning set with this procedure and were assessed postoperatively for their ability to learn new visual discriminations with the same procedure. Bilateral amygdalectomy did not significantly impair the animals' learning ability in this task. Learning remained unimpaired when transection of the uncinate fascicle and of the fornix was added to amygdalectomy. The effect of bilateral amygdalectomy in this task was much less severe than in a similar task we previously studied, with auditory secondary reinforcers. The results show that the involvement of the amygdala in processes of secondary reinforcement depends on the sensory properties of the secondary reinforcer. From these and other recent results, we conclude that the sensory attributes of a reinforcer are easily associated with a discriminative stimulus when they are in the same modality and same spatial location as the discriminative stimulus and that this sensory-sensory association is independent of the amygdala.
\end{abstract}

Bilateral amygdalectomy produces some well-known changes in monkeys. The animals show reduced emotional reactions both to attractive stimuli such as familiar foods and to aversive stimuli such as the net with which they have been caught (Weiskrantz, 1956; Gaffan and Harrison, 1987). In somc cxperimental cognitive tasks, amygdalectomy reduced performance and potentiated the effects of other lesions (Mishkin, 1978; Murray and Mishkin, 1985). Presumably, all these effects are functionally related to each other. In 2 recent experiments and in the present experiment, we have examined the involvement of the

\footnotetext{
Received Mar. 22, 1988; revised July 11, 1988; accepted July 13, 1988.

This research was supported by the Medical Research Council. We thank Dr. E. A. Murray for comments on an earlier draft.

Correspondence should be addressed to D. Gaffan, Department of Experimental Psychology, South Parks Road, Oxford OX1 3UD, England, or to E. A. Gaffan, Department of Psychology, Reading University, Whiteknights, Reading RG6 2AL, England.

Copyright @ 1989 Society for Neuroscience $0270-6474 / 89 / 020558-07 \$ 02.00 / 0$
}

amygdala in various aspects of the behavioral effects of food reward. Many experimental lasks are motivated by food reward, and food is also one of the natural stimuli to which amygdalectomized monkeys show abnormal emotional reactions.

The delivery of food reward in an experimental task is a complex event, possessing 3 different types of attribute which may be separately processed in the brain. (1) The food has an intrinsic incentive value because of its primary reinforcing properties, such as its taste and its nutritive value. (2) The reward event has some intrinsically neutral sensory properties, such as the click of a dispenser or the visual appearance of a piece of food. (3) The intrinsically neutral features of the event evoke some reactions from the animal because of the association in the animal's memory between those neutral features and the intrinsically rewarding features. When a stimulus is paired with food reward, the stimulus may be associated with any or all of these 3 types of attribute of the food reward event. If the 3 types of attribute are separately processed in the brain, the 3 types of association may be separately stored.

To investigate the role of the amygdala in learning for food reward, one must use tasks that assess separately the contributions of these 3 types of attribute of the food reward event. For this purpose the Wisconsin General Test Apparatus, the apparatus most frequently used in studies of primate learning and memory, has some disadvantages. In that apparatus a monkey displaces a visual stimulus object and finds a piece of food directly underneath it if the chosen object is the correct one. The correct object may enter into association with the intrinsically reinforcing attributes of the piece of food, and with incidental features such as its appearance or feel, and with the emotional reactions aroused by these incidental features. However, it is difficult to vary the contribution of these types of learning in this apparatus systematically. Therefore, in recent cxperiments described below and in the presently reported experiment, we have used an apparatus in which we are able to emphasize selectively one or another attribute of the reinforcer. In this apparatus, 2-dimensional visual stimuli are displayed on a touch-sensitive screen and the animal chooses between them by touching one. Visual learning can be reinforced either with food (the primary reinforcer) or with learned secondary reinforcers; secondary reinforcers do not possess the first type of attribute of a food reward, namely, its intrinsic incentive value. Furthermore, the sensory properties of the secondary reinforcers can be precisely controlled, in order to vary the relative contributions of the second and third types of attribute. The present experiment is concerned with the sensory properties of secondary reinforcers. 
E. A. Gaffan et al. (1988) examined visual learning for primary food reward. There was no visual feedback local to the chosen stimulus to indicate whether the choice was correct or not; instead, food rewards were delivered in a hopper beneath the stimulus display screen. Learning rate in this task was severely impaired by disconnection of the amygdala from the visual association cortex. [D. Gaffan et al. (1986) and E. A. Gaffan et al. (1986) had shown that bilateral lesions of visual association cortex impair learning in this task; similarly, in recent unpublished experiments, we have observed severe impairments following bilateral amygdalectomy in this task.] Gaffan and Harrison (1987) studied a different task in the same apparatus, visual discrimination learning for an auditory secondary reinforcer. Visual stimuli displayed on a screen were associated not with food but with an auditory secondary reinforcer, which itself was associated with food. Visual learning in this task was unaffected by disconnection of the amygdala from visual input; it was impaired, however, both by bilateral amygdalectomy and by disconnection of the amygdala from auditory input. As further discussed below and by Gaffan and Harrison (1988), these findings suggest that the role of the amygdala in this task was to sustain the auditory secondary reinforcer's association with the incentive value of the primary reinforcer. In the task used by E. A. Gaffan et al. (1988), the visual stimuli can themselves enter into association with the incentive value of food. Therefore, a coherent explanation for the findings so far is that the amygdala is specifically important for associating stimuli with the intrinsic incentive value of a food reward, the first of the types of attribute enumerated above. The relative importance of the second and third types of attributes can be manipulated by varying the sensory properties of a secondary reinforcer, as described below.

When a stimulus is paired with a secondary reinforcer (which has no intrinsic incentive value), the stimulus can be associated with the sensory properties of the secondary reinforcer and with the responses those properties evoke by virtue of their own association with the intrinsic incentive value of the primary reinforcer. Experiments with normal animals have shown that when the stimulus is in the same sensory modality as the secondary reinforcer, and is spatially contiguous with it, the stimulus is strongly associated with the sensory properties of the reinforcer; conversely, when the stimulus is in a different sensory modality from the secondary reinforcer, and spatially separated from it, such sensory-sensory associations are difficult to form and the stimulus is associated, instead, with the reactions evoked by the reinforcer (Rescorla, 1980, 1985). We assume that these generalizations apply to the intrinsically neutral sensory properties of primary and secondary reinforcers in our experiments. Both of the tasks in our 2 recent experiments described above were designed, on the basis of this assumption, to minimize the importance of associations between the visual discriminative stimuli and the sensory properties of the reinforcers. The auditory secondary reinforcers used by Gaffan and Harrison (1987) were delivered from a loudspeaker above the visual display screen, and the food reinforcers used by E. A. Gaffan et al. (1988) were delivered into a hopper below the screen, in a darkened room. Both these reinforcers were nonvisual and were spatially separate from the visual discriminative stimuli.

In the present experiment, the secondary reinforcer we used was visual and was spatially contiguous with the visual discriminative stimuli it was to be associated with. This procedure should facilitate the formation of visual-visual associations be- tween the discriminative stimuli and the intrinsically neutral sensory properties of the secondary reinforcer. If such associations are formed independently of the amygdala, one should expect relatively little effect of bilateral amygdalectomy in this task.

In the present task, the monkeys learned visual discriminations between pairs of stimuli displayed on a touch-sensitive screen, each new pair of stimuli constituting a new visual discrimination problem. If the negative stimulus of a pair was touched, it immediately disappeared. If the positive stimulus was touched, it remained on the screen and a white line immediately appeared over it. After a series of correct choices, food reward was delivered. If there was a series of incorrect choices, no reward was delivered and the opportunity for reward was lost. Thus, the white line was a visual secondary reinforcer, and immediate blanking of the screen was a visual secondary nonreinforcer. The task was the same as in Gaffan and Harrison's (1987) experiment, except that these events were visual instead of auditory. (In Gaffan and Harrison's experiment, the visual feedback on the screen was the same for correct and incorrect choices.) In this task, food is not given until a new discrimination problem is solved; thus, within-problem learning cannot reflect the acquisition of associations between the discriminative stimuli and food.

We first examined the effect of bilateral amygdalectomy, and compared it with the effect of bilateral amygdalectomy in the earlier task with auditory secondary reinforcers. Subsequently, we added 2 more ablations to the amygdalectomies. First, we cut the uncinate fascicle, then the fornix. The uncinate fascicle is potentially important in the neuropathology of human amnesia (Horel, 1978). This fascicle is the route of corticocortical interaction between the frontal lobe and the visual association cortex of the inferotemporal area (L. G. Ungerleider and D. Gaffan, unpublished observations). Behavioral experiments (in progress) suggest that it is important in certain kinds of visual conditional discriminations. The fornix is also important in some kinds of visual learning (Gaffan and Harrison, 1988). The combination of bilateral amygdalectomy with bilateral uncinate fascicle and fornix transection isolates the visual association cortex from many of the higher centers with which it normally interacts. However, if the present task reflects visual-visual associative learning, it may be that all the information entering into within-problem associative learning is present within the visual association cortex itsclf. If so, learning should be unaffected even after this sequence of surgeries.

\section{Materials and Methods}

\section{Subjects}

Three experimentally naive male cynomolgus monkeys (Macaca fascicularis) served in all stages of the experiment. At the time of their first surgery (sec below), they weighed on averagc $4.3 \mathrm{~kg}$. Beforc beginning the experimental task described below, they were first shaped to touch patterns displayed on the television screen, in the same way as described by Gaffan et al. (1984). They then received 40 sessions of training in 2-choice visual discrimination problems for immediate food reward, with the apparatus and stimuli described below. [This was the same pretraining in learning for immediate food reward as the animals had in the experiment of Gaffan and Harrison (1987).] Immediately afterwards they transferred to the present experimental task.

\section{Surgery}

After completing preoperative training, each animal was operated in 3 stages, with behavioral testing after each stage. The surgeries were carried out in aseptic conditions, with the aid of an operating microscope. 

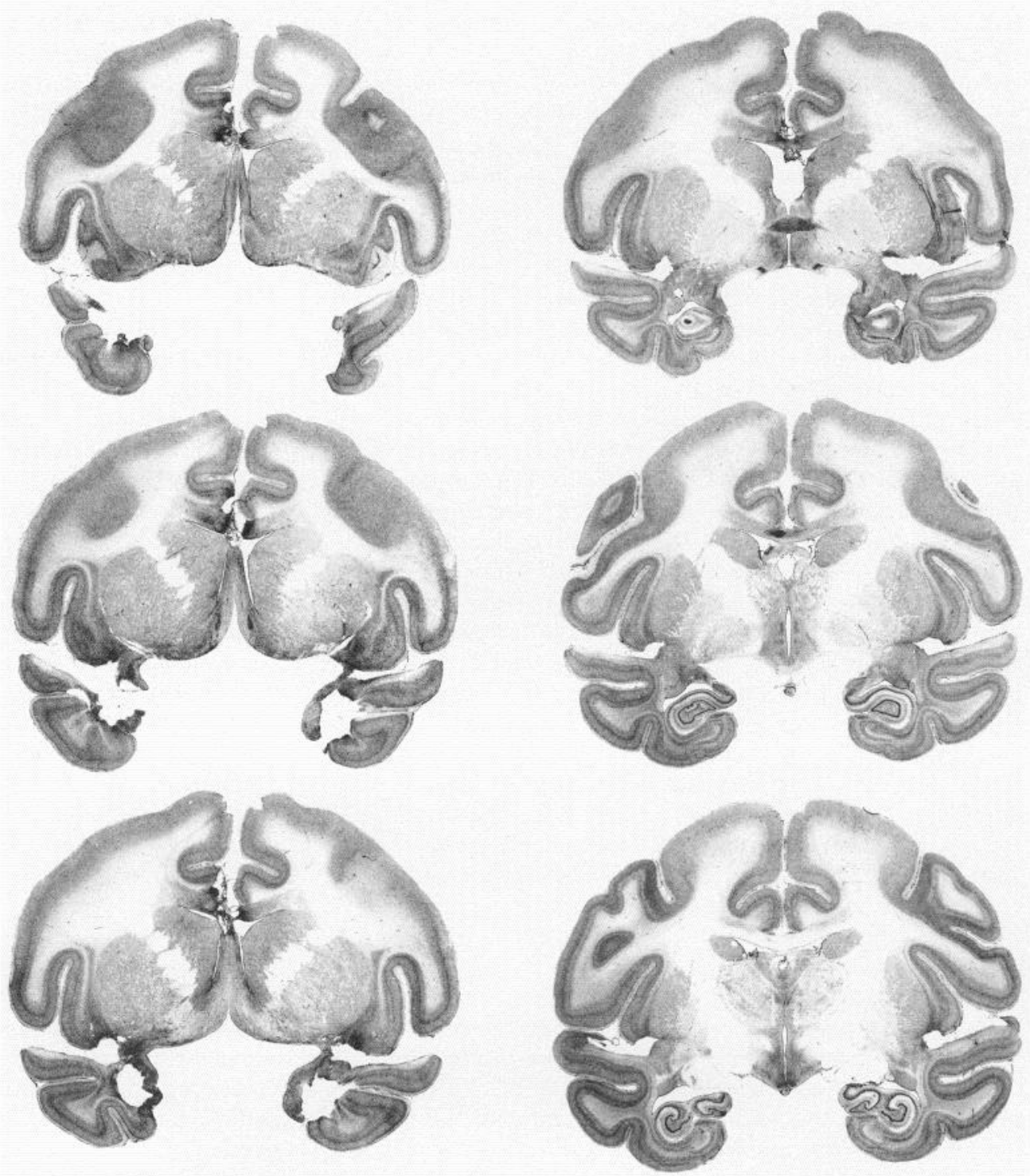

Figure 1. Series of sections, $1.25 \mathrm{~mm}$ apart, through the lesions in the brain of one of the subjects (this was the subject with the best individual performance level, before and after surgery, in the individual results shown in Fig. 4 , below). The most anterior section is at $t o p$ left, and the most posterior, bottom right.
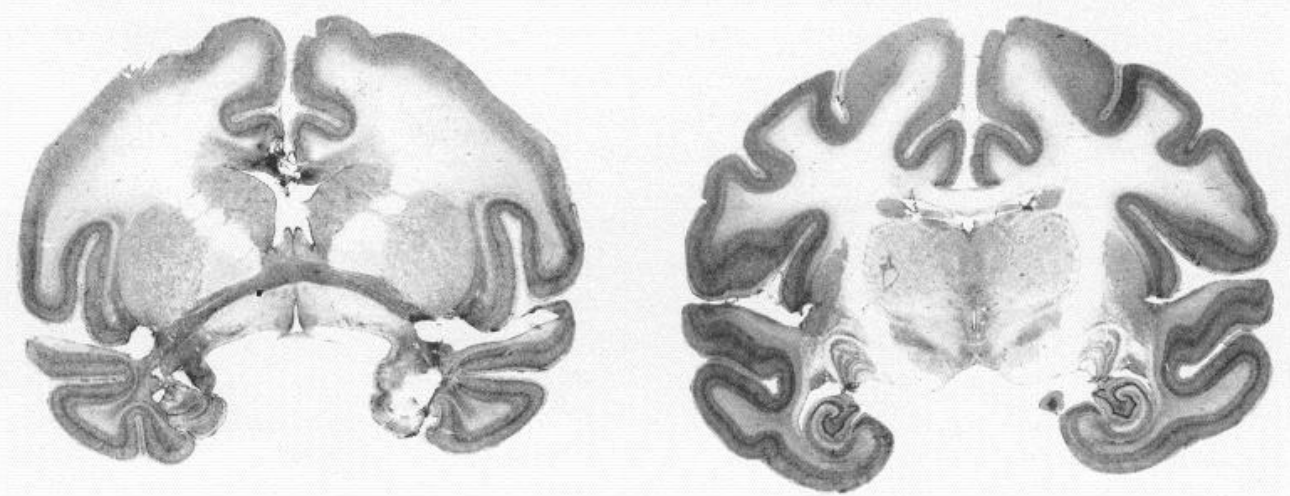

Anesthesia was maintained by barbiturate (thiopentone sodium B. P.) administered as necessary through an intravenous cannula. Following each stage of surgery, the animals were allowed a recovery period of $17-21 \mathrm{~d}$ before behavioral testing resumed.

Bilateral amygdalectomy. The amygdala was removed in the same way as in the experiments of Gaffan and Harrison (1987) and E. A. Gaffan et al. (1988). A craniotomy was opened over the frontal lobe and was extended with rongeurs anteriorly to the brow, medially within 2-3 mm of the midline, posteriorly to the level of the arcuate sulcus, and laterally to the level of the lateral sulcus. The dura was cut along the superior, posterior, and inferior margins of the craniotomy and turned back in a flap over the orbit and brow. The frontal lobe was gently retracted with a brain spoon to expose the medial surface of the anterior part of the temporal lobe. A 20 -gauge metal aspirator insulated except at the tip was used to cauterize and remove a patch of pia mater, 2-3 mm wide, on the medial surface of the temporal lobe superior and posterior to the tip of the rhinal sulcus. The amygdala was then ablated through the defect in the pia mater, by aspiration with the same metal aspirator. The lateral ventricle and the anterior surface of the hippocampus were visible posterior limits of the lesion. Laterally, gray matter 
of the amygdala was removed until white matter of the temporal stalk and uncinate fascicle appeared. Inferiorly and anteriorly the intention was to leave perirhinal and polar cortex intact, as far as possible. This procedure was carried out in both hemispheres successively in one operation. The dura was sewn, and the wound was closed in layers.

Bilateral section of the uncinate fascicle. In each hemisphere, a bone flap was raised over the lateral sulcus, extending from the orbit to the posterior tip of the lateral sulcus. The dura was cut to expose the lateral sulcus. The pia mater was cauterized along the inferior lip of the sulcus. Cortex in the inferior bank of the sulcus was removed by aspiration, from the temporal pole to the level of the tip of the central sulcus. Large vessels crossing the lips of the lateral sulcus were spared. The inferior part of the insula was lifted with a brain spoon to expose the underlying white matter. The white matter of the extreme and external capsules and uncinate fascicle was cut with cauterization and aspiration, until the lateral surface of the putamen was exposed. In the superior-inferior dimension the cut was made as narrow as possible in order to spare as much as possible of the insula and claustrum. In the anterior-posterior dimension the cut extended from the temporal pole to the level of the central sulcus. The dura was sewn, the bone flap was replaced, and the wound was closed in layers.

Bilateral fornix transection. A bone flap was raised over the midline of the skull. The dura was cut over the left hemisphere and retracted over the right hemisphere to expose the midline. Veins draining into the sagittal sinus were cauterized and cut in order to allow the left hemisphere to be retracted with a brain spoon. To expose the fornix the corpus callosum was cut with a glass aspirator in the midline in the region of the interventricular foramen, the cut extending $5-10 \mathrm{~mm}$ in the anterior-posterior direction. The fornix was then cauterized and cut with a metal aspirator. The dura was not sewn but was drawn back over the left hemisphere. The bone flap was replaced and the wound was closed in layers.

\section{Histology}

The animals were deeply anesthetized and then perfused through the heart with saline followed by formol-saline solution. The brains were blocked in the coronal stereotaxic plane posterior to the lunate sulcus and were then removed from the head, photographed, and allowed to sink in a sucrose formalin solution. The brains were cut in $50 \mu \mathrm{m}$ sections on a freezing microtome. Every fifth section was retained and stained with cresyl violet. The series of sections from each animal was examined microscopically. Illustrative sections from one monkey are shown in Figure 1. The other 2 monkeys were similar.

The amygdalectomies were similar to those in our previous experiments with this lesion (Gaffan and Harrison, 1987; E. A. Gaffan et al., 1988). Damage to the hippocampus was confined to the anterior $1-2$ $\mathrm{mm}$ of the hippocampus. Damage to the uncinate fascicle made during the course of amygdalectomy was impossible to assess in the present animals because of the subsequent intentional section of the uncinate fascicle. The annygdalectomies were in all cases complete except for some sparing of the dorsal posterior amygdala; the extent of sparing was similar to that in our previous experiments.

I' The transections of the uncinate fascicle were complete. Figure 2 shows the typical extent of the transection, as drawn onto the lateral surface of the brain. Cortex in the lower bank of the lateral sulcus was removed over the same anterior-posterior extent as the uncinate fascicle section. There was no evidence of damage to the overlying frontal and parietal lobes. In every case, the transection produced slight damage to the lateral ventral putamen and to the ventral claustrum, similar to that illustrated in Figure 1. Anteriorly, there was damage to the anterior commissure at the point where it leaves the anterior temporal lobe and separates from the uncinate fascicle.

The fornix transections were complete in all cases.

\section{Apparatus and stimuli}

The computer-controlled apparatus, described in detail by Gaffan et al. (1984), displayed pairs of complex patterns against a plain gray background on a television screen. The stimuli were presented $90 \mathrm{~mm}$ to the left and right of the screen's center. The monkey reached out through the bars of a transport cage to touch stimuli on the screen. Infra-red beams crossing the surface of the screen detected the animal's touches. Each of the 2 visual stimuli which constituted a discrimination problem was made of a small shape superimposed on a larger shape (approximate height 20 and $40 \mathrm{~mm}$, respectively). Each of the shapes was taken at

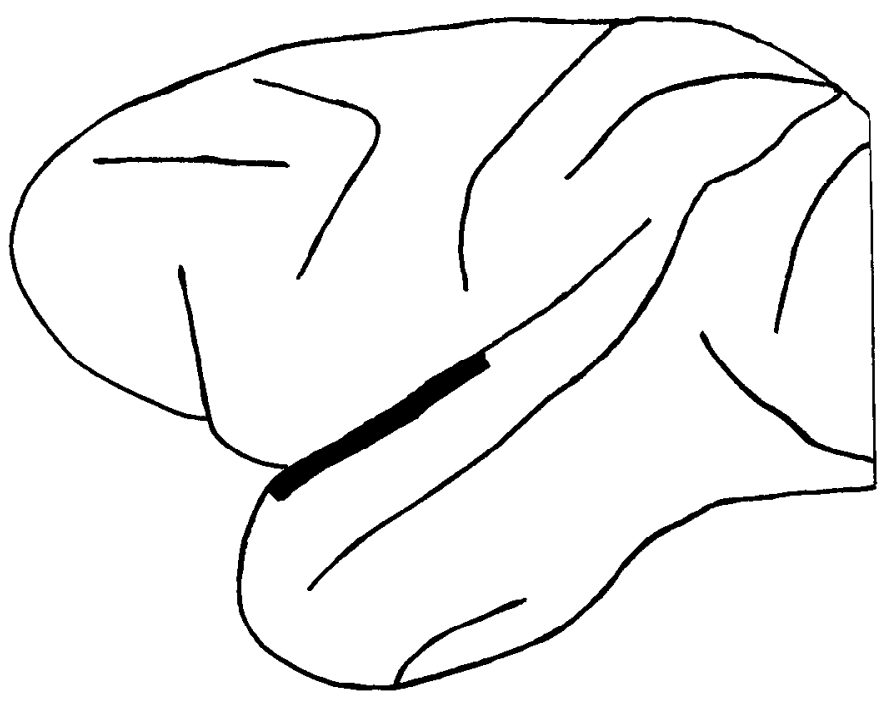

Figure 2. Extent of the uncinate fascicle section, made in the second surgical stage of the experiment, is shown as drawn onto the lateral surface of the brain. The lesion is shown as a widening of the anterior part of the lateral sulcus. This is how the lesion appears in photographs because of the ablation of the lower bank of the lateral sulcus that is made in the course of exposing the uncinate fascicle. The left hemisphere is shown, but all subjects had this lesion made in the right hemisphere also.

random from a predefined set of 127 symbols and was displayed in a color chosen at random from 255 possibilities. The random-number generator that determined stimuli for each problem and their left-right position at every trial was seeded with the session number, so the stimulus sequence was unique to each session but the same, in a given numbered session, for all monkeys.

Food rewards were dispensed into a bowl just in front of the lower edge of the screen. Each reward consisted of 2 pieces of food, either peanuts or sugar-coated puffed rice grains. The automatic dispenser made a $0.35 \mathrm{sec}$ buzz when it operated. The only visible illumination in the experimental cubicle came from the television screen. The background color of the screen, against which stimuli were displayed, was a midgray that shed enough light to see by, but this was blacked out when $10 \mathrm{sec}$ of darkness was required by the training schedule as described below. An infra-red light source allowed the monkey to be watched over closed-circuit television.

\section{Main task}

Each daily session had 50 new visual discrimination problems, presented consecutively, with an interval between problems of $10 \mathrm{sec}$. For each new problem, a positive and a negative stimulus were chosen at random from the large population of available stimuli (see Apparatus). For each trial within a problem, the 2 stimuli were presented side by side, occupying the left and right positions at random, and the animal chose one stimulus by touching it. If the chosen stimulus was the negative, both stimuli immediately disappeared from the screen. If the chosen stimulus was the positive, the negative stimulus immediately disappeared, and, at the same time, a vertical white line $1 \mathrm{~mm}$ thick and extending the full height of the screen appeared over the center of the positive stimulus. The positive stimulus and the white line disappeared together $0.5 \mathrm{sec}$ later. The intertrial interval, measured from the animal's touching the stimulus, was $4 \mathrm{sec}$. Trials continued in this fashion until either the negative or the positive stimulus was chosen consistently for 4 trials consecutively. If the positive stimulus was chosen 4 times consecutively, a reward was dispensed at the end of the fourth white-line display. If the negative stimulus was chosen 4 times consecutively, the fourth choice immediately produced $10 \mathrm{sec}$ darkness. Either of these events, the primary reinforcer and the primary nonreinforcer, terminated the current problem. The number of trials per problem was thus not fixed but was always at least 4 , enabling learning curves to be drawn for trials $1-4$. 
Figure 3. Group learning curves are shown for each of the 4 stages of the experiment.

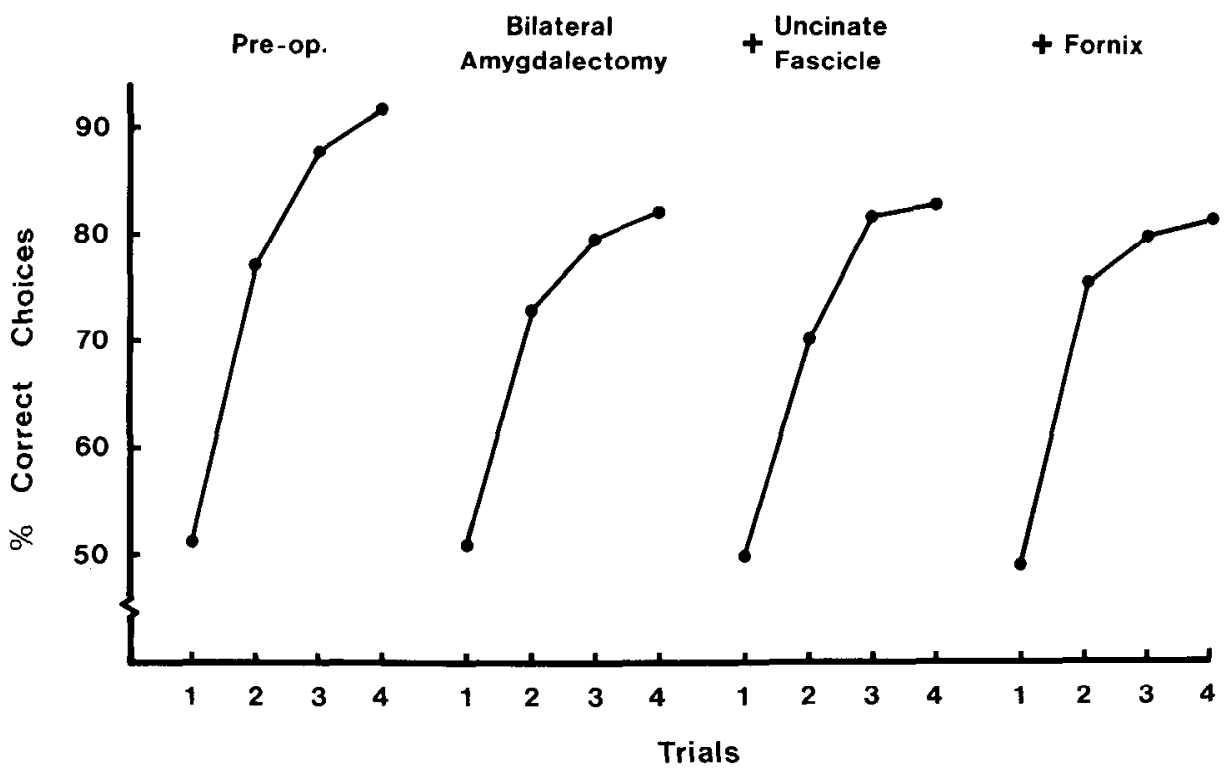

Postoperative training

Following each of the 3 surgical operations, each animal worked at the main task for 20 sessions.

After $3 \mathrm{~d}$ of the projected $20 \mathrm{~d}$ testing period following uncinate fascicle section, the experiment had to be suspended for $48 \mathrm{~d}$ because of a salmonella infection in the monkey colony. None of the present subjects was infected, but a quarantine was imposed on the whole colony in order to curb the spread of the infection. When testing was resumed, a further 20 sessions were given.

\section{Results}

The learning curves in Figure 3 show the performance of the animals during the first 4 trials of new discrimination problems, averaged across the last 5 sessions of the 20 session test phase at each stage of the experiment. By the end of preoperative training, all subjects were showing rapid within-problem learning. Following bilateral amygdalectomy, they continued to acquire new problems without difficulty, although there was a small deterioration on average in the rate of learning. Neither transection of the uncinate fascicle nor fornix transection produced any further impairment.

To assess the statistical significance of the effect of amygdalectomy, we analyzed error rates on trial 4 of new problems presented in the last 5 sessions before operation and the last 5 sessions of the test following amygdalectomy. This measure is the same as that which we used in an earlier experiment with auditory secondary reinforcers (Gaffan and Harrison, 1987). In both tasks, all problems were presented for a minimum of 4 trials before any primary reinforcement, as described in $\mathrm{Ma}-$ terials and Methods above. Because the present experiment was performed in the same say as the earlier experiment, the results of bilateral amygdalectomy can be directly compared and analyzed together. The results are shown in this form in Figure 4. Preoperative performance at the 2 tasks was similar, the group trained with the visual reinforcer being slightly but not significantly better $[t(4)=1.21, p>0.10]$. The postoperative deficit following amygdalectomy, as assessed by the drop in trial 4 performance, was significantly greater in the task with auditory secondary reinforcers than in the present task $[t(4)=2.32, p<$ $0.05]$. The same measure, the drop in trial 4 performance, was
Figure 4. Effects of bilateral amygdalectomy in the present experiment (visual secondary reinforcement) are compared with its effects in a similar earlier experiment, in which auditory secondary reinforcers were used (Gaffan and Harrison, 1987). The bars show the group means, and the dots, connected by lines, show the performance of individual monkeys. 
used in planned comparisons (using the pooled within-group error estimate) to assess the effects of amygdalectomy in the 2 tasks separately. The drop in trial 4 performance was significant (i.e., $>0$ ) in the task with auditory reinforcement $[t(4)=3.79$, $p<0.05$ ], but the drop in the present task did not attain statistical significance $[t(4)=1.47, p>0.10]$.

Figure 4 shows the results of individual animals. It can be seen that among the 3 animals in the present experiment, the drop in trial 4 performance following amygdalectomy varied between 2 and 14\%. The brain illustrated in Figure 1 is from the monkey that showed the drop of only $2 \%$. There is no indication that this animal's bilateral amygdalectomy was less complete than those of the animals in Gaffan and Harrison's experiment (1987). Also, the 3 individuals in the present experiment had similar lesions, with comparable sparing of the dorsal posterior amygdala (see Histology, above). Thus, variation between individuals in the extent of the amygdalectomy does not appear to be an important factor in explaining either the difference between the tasks in sensitivity to amygdalectomy or the variance between individuals within a task.

\section{Discussion}

Bilateral amygdalectomy had little effect upon the animals' ability to learn visual discrimination problems for a visual secondary reinforcer that was spatially contiguous with the positive visual discriminative stimulus. Learning rates in this task declined slightly, on average, after bilateral amygdalectomy, but the decline did not attain statistical significance. Most importantly, the decline observed in the present task was significantly less than the decline we previously observed following bilateral amygdalectomy in a task similar to the present task, but using auditory secondary reinforcers (Gaffan and Harrison, 1987).

In the introduction, we distinguished 3 types of attributes of a food reward delivery: its intrinsic incentive value, its intrinsically neutral sensory features, and the reactions that the neutral features evoke in the animal because of their association in memory with the intrinsic incentive value. The learned auditory and visual secondary reinforcers we have used are similar to each other in lacking intrinsic incentive value (the first of these types of attribute). Also, they are both associated in memory with primary reinforcement and in the normal animal evoke reactions (the third type of attribute) because of this association. The difference between them is in their intrinsically neutral sensory properties (the sccond type of attributc). One of them is in the same modality as the discriminative stimuli and in the same spatial location, the other is not. For this reason, as explained in the introduction, associations between the discriminative stimuli and the sensory features of the secondary reinforcer may be expected to be facilitated by the visual secondary reinforcer, as compared with the auditory secondary reinforcer. Therefore, from the fact that bilateral amygdalectomy had far less effect on learning for the visual than for the auditory secondary reinforcer (Fig. 4), we conclude that the sensory-sensory association, between a visual discriminative stimulus and the visual properties of a spatially contiguous reinforcer, is independent of the amygdala.

Our overall hypothesis is that the amygdala is specifically important for storing associations with the intrinsic incentive value of a reward event, and not with other attributes of the event. In experiments with secondary reinforcers, this type of association is formed between the sensory properties of the secondary reinforcer and the incentive value of the primary rein- forcer. Once formed, that association sustains the secondary reinforcer's power to evoke emotional and other reactions, the third type of attribute of a reward event enumerated above. When, as in the 2 tasks considered above, a visual discriminative stimulus is followed by a secondary reinforcer, there are 2 possible routes for discriminative learning: The discriminative stimulus may enter into association either with the emotional reactions aroused by the secondary reinforcer or with the reinforcer's sensory properties. The amygdala is important for mediating the learned emotional reactions to the secondary reinforcer. Therefore, when discriminative learning is dependent on the first of these 2 processes (as in the task of Gaffan and Harrison, 1987), amygdalectomy produces a large impairment, but when the second, sensory-sensory type of association predominates (as in the present task), this surgery has less effect.

This overall hypothesis offers a parsimonious explanation of the effects we have observed both with secondary and with primary reinforcers. Disconnection of the amygdala from visual association cortex impaired visual discrimination learning for primary reinforcement (E. A. Gaffan et al., 1988) but did not impair visual discrimination learning for auditory secondary reinforcement (Gaffan and Harrison, 1987). Thus, the interaction of visual association cortex with the amygdala is not necessary when, as the above analysis suggests, visual stimuli are associated principally with the reactions a reinforcer evokes, but it is necessary when the normal animal can directly associate visual stimuli with the reinforcer's intrinsic incentive value.

The samc hypothesis can also account for the informally observed changes in emotional reactions, which have been reported to follow amygdala lesions. In the normal animal, excitement at the sight of food or fear at the sight of a net is elicited by the associations of those visual stimuli with the intrinsic incentive or disincentive value of having food in the mouth or being confined in the net. Thus, a lesion that interfered with those associations would produce the observed decrements in those reactions.

The present results are relevant to the interpretation of behavioral effects of amygdalectomy in the Wisconsin General Test Apparatus, in which many previous investigations of amygdalar function have been carried out. In that apparatus, a monkey displaces a visual stimulus object and, if the chosen object is the correct one, finds a piece of food directly underneath it. As a result of this event, the animal might remember a sensory association between the visual discriminandum and the visual properties of the reward underneath it. Monkeys can rapidly learn associations between displayed stimuli and the visual properties of non-food objects found under the displaced stimuli (Gaffan and Bolton, 1983). It is possible therefore that the same type of association might be formed between a displaced stimulus and the visual properties (as distinct from the incentive value) of a piece of food found underneath it. Our present results imply that that type of association is not dependent on the amygdala. This could explain why amygdalectomy produces variable and sometimes quite mild effects upon visual discrimination learning for food reward in the Wisconsin General Test Apparatus. [Gaffan and Harrison (1987) briefly review and discuss the results from this apparatus.]

Murray and Mishkin (1985) observed severe effects of bilateral amygdalectomy in a cross-modal task in which animals had to match the feel of an object with the sight of the object. They suggested that the amygdala might be specifically important for cross-modal sensory associations. Their hypothesis accommo- 
dates well the present results since the task of the present expcriment provided a strong within-modal association. Also, their hypothesis is consistent with the effects of bilateral amygdalectomy in Gaffan and Harrison's (1987) experiment, with auditory secondary reinforcers; these auditory stimuli, and the reactions they evoked because of their association with primary reinforcement, were all nonvisual events. However, their hypothesis cannot readily explain the effects of disconnection in Gaffan and Harrison's experiment. In that experiment, visual cortex was isolated from the amygdala without any decrement in learning ability. We conclude from this that the cross-modal associations of visual stimuli with the reactions to the secondary reinforcer were not dependent on the interaction of the visual cortex with the amygdala. Instead, a specific subset of cross-modal associations, i.e., the association of the auditory secondary reinforcer with the incentive value of the primary reward, was dependent on the amygdala in that experiment.

It is much less clear how the present hypothesis should be applied to Murray and Mishkin's results. Those results were obtained in the Wisconsin General Test Apparatus, and the severe impairment observed in their cross-modal task was contrasted with milder impairments in within-modal tasks in that apparatus. We have suggested that the Wisconsin General Test Apparatus facilitates within-modal associations of discriminative stimuli with motivationally neutral sensory features of the food reward. It is possible that cross-modal tasks in that apparatus cannot rely so heavily on this type of association because neither the tactile nor the visual properties of the food reward event can enter into strong within-modal associations with both of the modalities of the discriminative stimulus. This is only a tentative suggestion, however. It is an important goal for future experiments, including disconnection experiments, to analyze the associative structure of food-motivated cross-modal tasks, including that introduced by Murray and Mishkin.

The second and third surgical stages of the experiment showed that the present type of learning remained unaffected even when transection of the uncinate fascicle and of the fornix was added to the amygdalectomy. Thus, this type of learning remains possible when the visual association cortex of the inferotemporal area is cut off from several of its main output pathways, through the hippocampal system and fornix, the amygdala, and the pro- jections to the frontal lobe via the uncinate fascicle. The inferotemporal area is relatively self-sufficient in visual-visual associative learning of the type we have studied in the present experiment. No doubt the monkey's visual-visual associative memory is richer than that studied here. Our present aim, however, was to study visual-visual associations in the context of simple visual discrimination learning, in which visual discriminative stimuli need to be associated with only 2 possible events, reward and nonreward.

\section{References}

Gaffan, D., and J. Bolton (1983) Learning of object-object associations by monkeys. Q. J. Exp. Psychol. 35B: 149-155.

Gaffan, D., and S. Harrison (1987) Amygdalectomy and disconnection in visual learning for auditory secondary reinforcement by monkeys. J. Neurosci. 7: 2285-2292.

Gaffan, D., and S. Harrison (1988) Place memory and scene memory: Effects of fornix transection in the monkey. Exp. Brain Res. (in press).

Gaffan, D., R. C. Saunders, E. A. Gaffan, S. Harrison, C. Shields, and M. J. Owen (1984) Effects of fornix transection upon associative memory in monkeys: Role of the hippocampus in learned action. $Q$. J. Exp. Psychol. 36B: 173-221.

Gaffan, D., S. Harrison, and E. A. Gaffan (1986) Visual identification following inferotemporal ablation in the monkey. Q. J. Exp. Psychol. 38B: $5-30$

Gaffan, E. A., S. Harrison, and D. Gaffan (1986) Single and concurrent discrimination learning by monkeys after lesions of inferotemporal cortex. Q. J. Exp. Psychol. 38B: 31-51.

Gaffan, E. A., D. Gaffan, and S. Harrison (1988) Disconnection of the amygdala from visual association cortex impairs visual reward-association learning in monkeys. J. Neurosci. 8: 3144-3150.

Horel, J. A. (1978) The neuroanatomy of amnesia: A critique of the hippocampal memory hypothesis. Brain 101: 403-445.

Mishkin, M. (1978) Memory in monkeys severely impaired by combined but not by separate removal of amygdala and hippocampus. Nature 273: 297-298.

Murray, E. A., and M. Mishkin (1985) Amygdalectomy impairs crossmodal association in monkeys. Science 228: 604-606.

Rescorla, R. A. (1980) Pavlovian Second-Order Conditioning: Studies in Associative Learning, Erlbaum, Hillsdale, NJ.

Rescorla, R. A. (1985) Associationism in animal learning. In Perspectives on Learning and Memory, L. Nilsson and T. Archer, eds., pp. 39-61, Erlbaum, Hillsdale, NJ.

Weiskrantz, L. (1956) Behavioral changes associated with ablation of the amygdaloid complex in monkeys. J. Comp. Physiol. Psychol. 49. $381-391$. 